\title{
Color Inference Using an Enhanced Fuzzy Method
}

\author{
Sang-Geol Lee*, Kwang-Baek Kim**, Eui-Young Cha* \\ *Dept. of Computer Engineering, Pusan National University, Busan, Korea \\ **Dept. of Computer Engineering, Silla University, Busan, Korea \\ leesg@pusan.ac.kr
}

\begin{abstract}
Color information recognition methods based on the RGB color model, which is designed on the basis of static fuzzy inference rules, are being widely used at present. However, these methods have certain limitations because of the nature of the model used: detachment of human vision and limited choice of environment. In this paper, we propose a method based on the HSI model and a new inference process that resembles the human vision recognition process. This method allows the user to add, delete, or update inference rules. In our method, membership intervals are designed with sine and cosine functions in the $H$ channel and trigonometric style functions in the $S$ and $I$ channels. The membership degree is computed via an interval merging process. Then, inference rules are applied to the result in order to infer the color information. Experimental results show that our method is more intuitive and efficient than that based on the RGB model.
\end{abstract}

Keywords : Color Information Recognition, Inference Rules, HSI Model, Membership Intervals

\section{Introduction}

The RGB color model is an additive model in which red, green, and blue light can be merged in different ways to reproduce a broad array of colors. Computer systems that show the human eyes perception of the light reflected from an object are designed on the basis of the RGB model. However, the vision recognition process in humans is different from that in the RGB model[6]. For example, a human recognizes and interprets the color red as "bright, saturate, is a red color line", as opposed to computer systems, which represent the color red by the digitized RGB $(255,0,0)$ value. Fuzzy theory enables perception and reasoning abilities similar to those of humans to be used in computer systems[2]. According to this theory, conventional color recognition methods based on the RGB model cannot be applied to real problems[3, 4].

In contrast, the color representation method in the HSI model is similar to human perception; in this model, fixed values of $\mathrm{H}(\mathrm{Hue}), \mathrm{S}$ (Saturation), and I(Intensity) are used to represent colors. When the color red is represented by $\mathrm{H}: 0^{\circ}, \mathrm{S}: 1.00$, and $\mathrm{I}: 255$, the implied meaning would be "bright, saturate, is a red line". Therefore, when the fuzzy theory is considered to be similar to human perception, it can be inferred that the color component will create the conditions[8]. In addition, in the HSI model, the inference rules can be added, modified, or deleted by users.

Applying new methods to existing fuzzy logic is inefficient because inference rules will have to be set for all possible combinations with pre-defined membership intervals. This implies that new inference rules added may act as inconvenience factors. For example, when new conditions are defined for fuzzy logic application, incorrect conclusions can be drawn; this is because of the fixed 
membership intervals and inference rules. In such a case, modification of the existing inference rules would be an efficient method, as the lifetime of an application is an important consideration in software engineering.

In a previously proposed method[3], in which color recognition was performed using the RGB model, diagnostic results were not obtained for new colors. In addition, not all colors could be analyzed and recognized on the basis of the membership intervals and fuzzy inference rules. However, in the present study, the proposed color inference method is easily applied with the same membership intervals to modify pre-defined inference rules so that they are relevant to the new conditions.

\section{Proposed method of color information analysis}

\subsection{Color inference using the HSI model}

The HSI model consists of three components: Hue(H), Saturation(S), and Intensity(I). In this paper, we calculate membership degrees in Saturation and Intensity using triangular fuzzy membership functions. Each of the membership intervals are defined in Tables 1 and 2.

Table 1. Saturation membership intervals

\begin{tabular}{ll}
\hline Interval (Abbreviation notation) & Boundary Value \\
\hline Very Low (VL) & {$[0,0.1]$} \\
Low $(\mathrm{L})$ & {$[0.05,0.56]$} \\
Normal $(\mathrm{N})$ & {$[0.45,1.0]$} \\
\hline
\end{tabular}

Table 2. Intensity membership intervals

\begin{tabular}{ll}
\hline Interval (Abbreviation notation) & Boundary Value \\
\hline Very Dark (VD) & {$[0,30]$} \\
Dark (D) & {$[20,135]$} \\
Bright (B) & {$[115,250]$} \\
Very Bright (VB) & {$[240,255]$} \\
\hline
\end{tabular}

Hue is defined as the angle corresponding to the indicated color on the color wheel (from $0^{\circ}$ to $360^{\circ}$ ). So membership intervals of Hue are calculated with the cosine function, as shown in Table 3.

Table 3. Hue membership intervals using the cosine function

\begin{tabular}{ll}
\hline Interval (Abbreviation notation) & Boundary Value \\
\hline Red (R) & {$\left[330^{\circ}, 30^{\circ}\right]$} \\
Yellow (Y) & {$\left[30^{\circ}, 90^{\circ}\right]$} \\
Green (G) & {$\left[90^{\circ}, 150^{\circ}\right]$} \\
Cyan (C) & {$\left[150^{\circ}, 210^{\circ}\right]$} \\
Blue (B) & {$\left[210^{\circ}, 270^{\circ}\right]$} \\
Magenta (M) & {$\left[270^{\circ}, 330^{\circ}\right]$} \\
\hline
\end{tabular}


However, there is a problem with membership intervals of intermediate colors. As shown in Figure. 1, the use of the cosine curve to find membership intervals is ambiguous for intermediate colors because of the clear boundaries between colors. Therefore, we added the sine function to Hue membership intervals, as shown in Table 4.

Table 4. Hue membership intervals using the sine function

\begin{tabular}{ll}
\hline Interval (Abbreviation notation) & Boundary Value \\
\hline Red-Yellow (R-Y) & {$\left[0^{\circ}, 60^{\circ}\right]$} \\
Yellow-Green (Y-G) & {$\left[60^{\circ}, 120^{\circ}\right]$} \\
Green-Cyan (G-C) & {$\left[120^{\circ}, 180^{\circ}\right]$} \\
Cyan-Blue (C-B) & {$\left[180^{\circ}, 240^{\circ}\right]$} \\
Blue-Magenta (B-M) & {$\left[240^{\circ}, 300^{\circ}\right]$} \\
Magenta-Red (M-R) & {$\left[300^{\circ}, 360^{\circ}\right]$} \\
\hline
\end{tabular}

As the sine and cosine curves have a one period for $0^{\circ} \sim 360^{\circ}$ and there is a negative value, the membership intervals $(f(x))$ of input value $(\theta)$ are calculated as follows:

$$
\begin{gathered}
f(x)=|\cos (3 \theta)| \\
f(x)=|\sin (3 \theta)|
\end{gathered}
$$

Figure 1 shows the membership function for membership intervals for a total of 12 different colors.

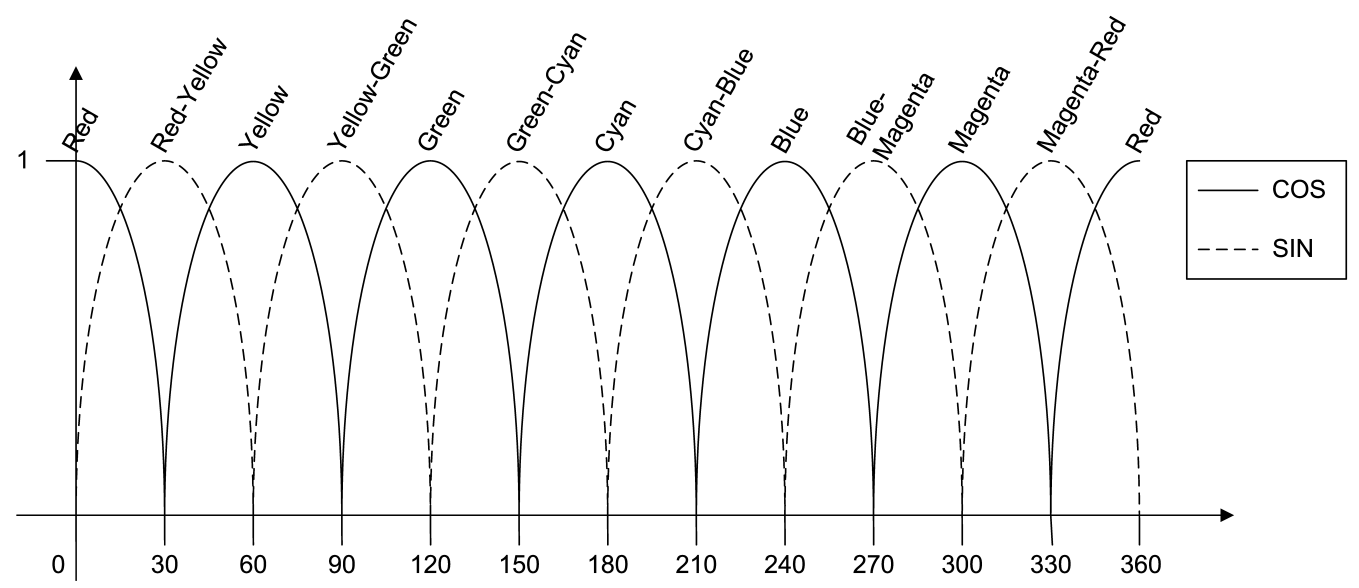

Figure 1. Hue membership function

\subsection{Eleven basic inference rules}

As in conventional methods, the proposed method has 11 basic inference rules, which are defined in Table 5. 
Table 5. Basic inference rules

\begin{tabular}{|c|c|}
\hline Condition & Conclusion \\
\hline If $\mathrm{i}=\mathrm{VD} \& \mathrm{~s}=\mathrm{X} \& \mathrm{~h}=\mathrm{X}$ & $\mathrm{r}=$ Black \\
\hline If $\mathrm{i}=\mathrm{D}|\mathrm{B}| \mathrm{VB} \& \mathrm{~s}=\mathrm{L} \mid \mathrm{N} \& \mathrm{~h}=\mathrm{Y}$ & $\mathrm{r}=$ Yellow \\
\hline If $\mathrm{i}=\mathrm{D}|\mathrm{B}| \mathrm{VB} \& \mathrm{~s}=\mathrm{L} \mid \mathrm{N} \& \mathrm{~h}=\mathrm{G}$ & $\mathrm{r}=$ Green \\
\hline If $\mathrm{i}=\mathrm{D}|\mathrm{B}| \mathrm{VB} \& \mathrm{~s}=\mathrm{L} \mid \mathrm{N} \& \mathrm{~h}=\mathrm{B}-\mathrm{M}$ & $\mathrm{r}=$ Purple \\
\hline If $\mathrm{i}=\mathrm{D}|\mathrm{B}| \mathrm{VB} \& \mathrm{~s}=\mathrm{L} \mid \mathrm{N} \& \mathrm{~h}=\mathrm{R}$ & $r=\operatorname{Red}$ \\
\hline If $\mathrm{i}=\mathrm{B} \mid \mathrm{VB} \& \mathrm{~s}=\mathrm{L} \& \mathrm{~h}=\mathrm{R}-\mathrm{Y}$ & $\mathrm{r}=$ Orange \\
\hline If $\mathrm{i}=\mathrm{B} \mid \mathrm{VB} \& \mathrm{~s}=\mathrm{L} \& \mathrm{~h}=\mathrm{R}$ & $r=$ Pink \\
\hline If $\mathrm{i}=\mathrm{D}|\mathrm{B}| \mathrm{VB} \& \mathrm{~s}=\mathrm{L} \mid \mathrm{N} \& \mathrm{~h}=\mathrm{B}$ & $r=$ Blue \\
\hline If $\mathrm{i}=\mathrm{D} \& \mathrm{~s}=\mathrm{N} \& \mathrm{~h}=\mathrm{R}-\mathrm{Y}$ & $\mathrm{r}=$ Brown \\
\hline If $\mathrm{i}=\mathrm{D} \mid \mathrm{B} \& \mathrm{~s}=\mathrm{VL} \& \mathrm{~h}=\mathrm{X}$ & $\mathrm{r}=$ Gray \\
\hline If $\mathrm{i}=\mathrm{VB} \& \mathrm{~s}=\mathrm{VL} \& \mathrm{~h}=\mathrm{X}$ & $\mathrm{r}=$ White \\
\hline
\end{tabular}

\subsection{Membership interval merger}

Conventional fuzzy methods require inference rules for all combinations that can be derived from of the pre-defined membership intervals[9]. This means that inference rules cannot be modified at runtime. In this paper, we do not combine inference rules for all membership intervals to modify inference rules at runtime. However, we solve the problem by merging unused membership intervals into used membership intervals so that the problem of unused intervals occurs.

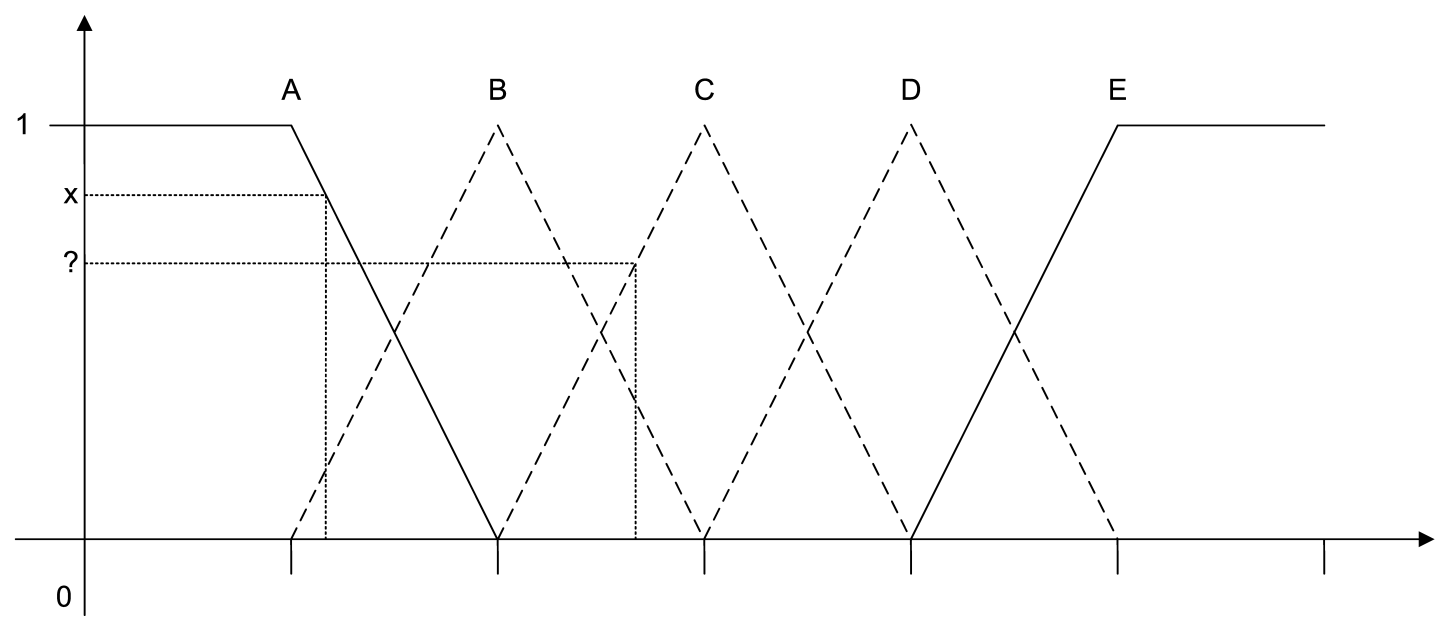

Figure 2. Membership function for $A$ and $C$ intervals

In Figure. 2, the membership function $f(x)$ has membership intervals of A, B, C, and D. Here, if only membership intervals $A$ and $E$ are used and intervals $B, C$, and $D$ are excluded, we can calculate the membership degrees of the input values on intervals $A$ and $E$, but lose the means of calculating the membership degrees of intervals B, C, and D. Therefore, a process is needed that merges the membership degrees of the input values of intervals $B, C$, and $D$ into intervals $A$ and $E$. In this paper, we propose an intersection point membership degree method.

As shown in Figure. 3, when the value of the $C$ interval is input, we can semantically draw a line from point $b$ to point $a$. Then the intersection point $r$ for the graph of membership degree $A$ occurs 


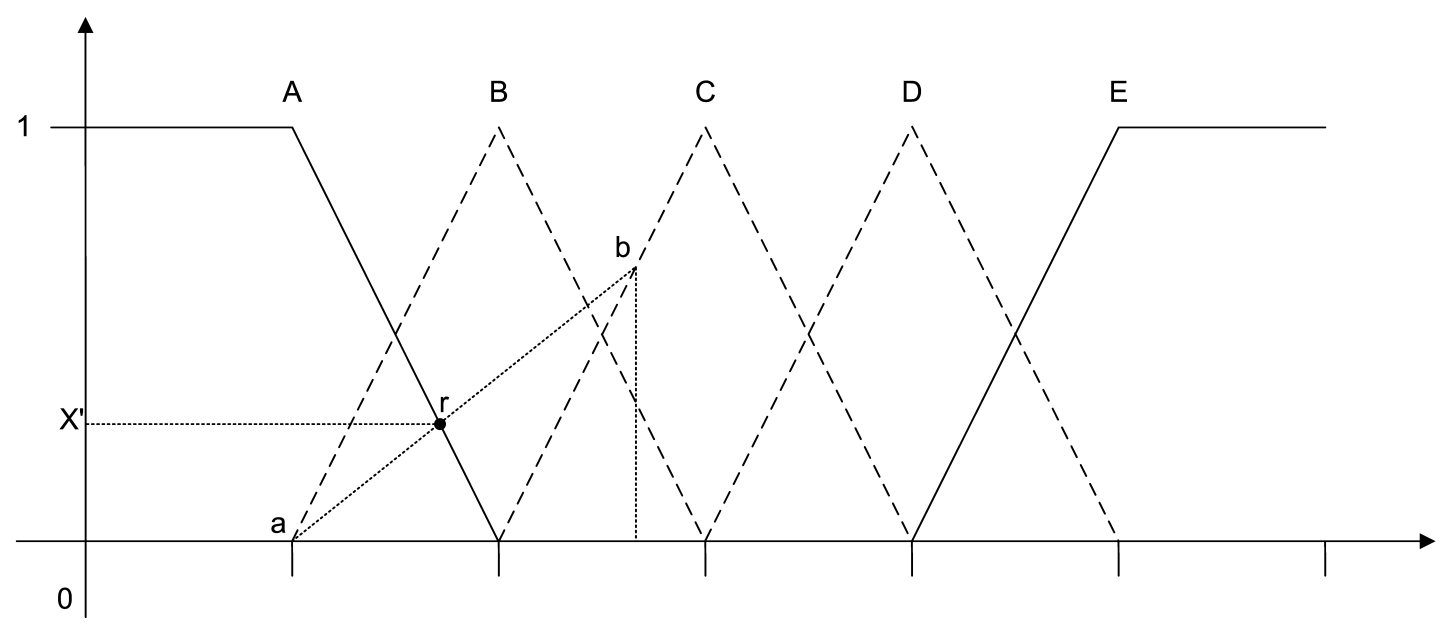

Figure 3. Merger of membership degree for the $C$ interval

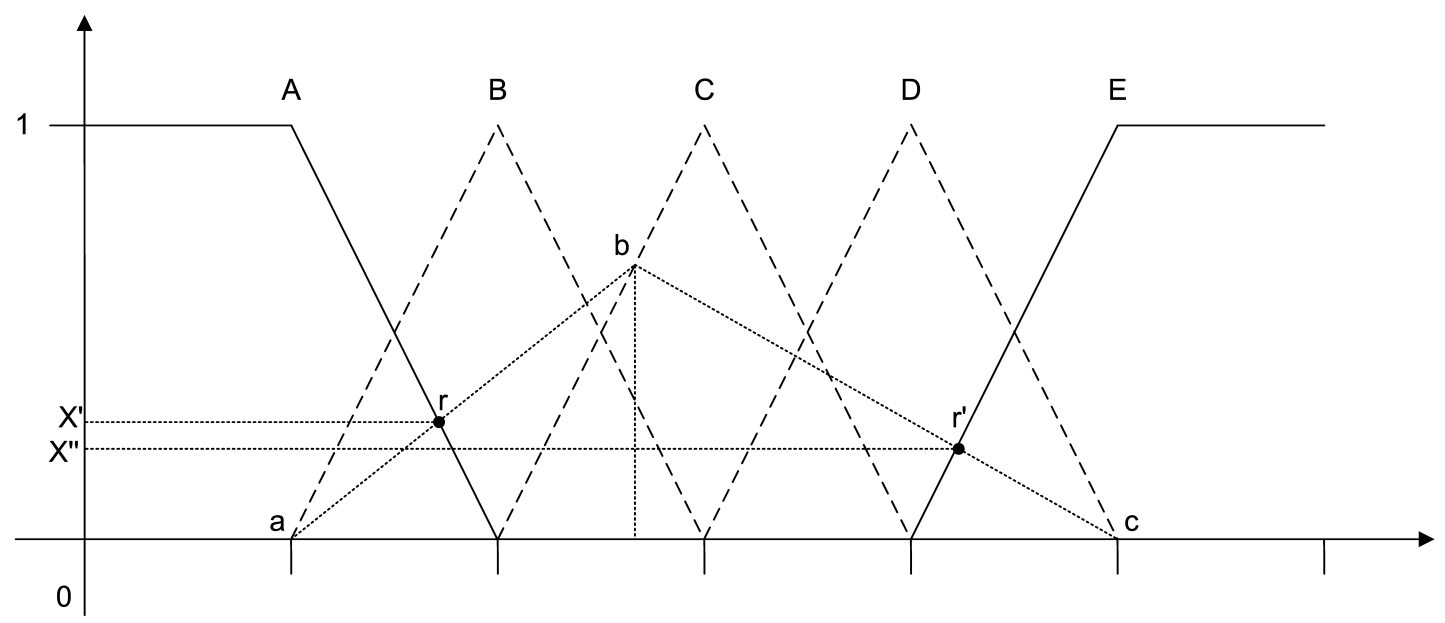

Figure 4. Membership degree $x^{\prime}$ and $x^{\prime \prime}$ of $r$ and $r^{\prime}$

and this point $(r)$ is used as the membership degree $\left(x^{\prime}\right)$ for merging interval $C$ into $A$.

As shown in Figure. 4, the intersection point $r^{\prime}\left(x^{\prime \prime}\right)$ occurs between points $b$ and $c$; this time, we select the interval corresponding to the Max value. In this way, we merge unused membership intervals. Another proposed method involves selection of the merging interval and the subsequent selection of an input value that is close to A or E. In this study, we use the latter method; experimental results show that this method is suitable for accurate color information recognition.

The reason for obtaining the intersection point is that it may cause a decrease in the confidence level; this is because the membership degree value of the y-axis pair of $b$ is not the membership degree value for $\mathrm{A}$, and the membership degree value is used in interval $\mathrm{C}$ only for close distances[1, 5]. This problem has been reported in the case of the Mamdani inference method[7, 10]. Thus, we can assign a certain weight to the intersection point that is obtained by admissions to some degree for the membership degree of interval $\mathrm{C}$, depending on the distance. By carrying out experiments, we prove that the results of consequence analyses are reliable. 


\subsection{Changing inference rules}

Finally, in this paper, we propose a method that adds, modifies, or deletes inference rules to adapt for variations in application conditions. Previously, in Table 5, we defined 11 inference rules. Subsequently, we added a twelfth inference rule and experimented.

This method to change inference rules can modify "bright $(244)$, light $(0.1)$, yellow line $\left(60^{\circ}\right)$ " to mean characters such as "bright(B), light(L), yellow line(Y)". In experiments, we analyzed the inference rules that are defined as Beige and confirmed a more intuitive, ergonomically efficient method to change conventional inference rules, while running human experiments in parallel.

\subsection{Processing for redundant inference rules}

Redundant inference rules occur when inference rules are added, modified, or deleted. In the cases of Red and Pink, the inference rules were analyzed, as shown in Table 6. These two colors have the same inference rules but with different intervals. We chose the method that processes a smaller number of inference rules because there is a higher possibility of a unique color when there is a smaller number of inference rules. In other words, we applied refined inference rules for the two colors, as shown in Table 7.

\begin{tabular}{|c|c|}
\hline Condition & Conclusion \\
\hline If $\mathrm{i}=\mathrm{D}|\mathrm{B}| \mathrm{VB} \& \mathrm{~s}=\mathrm{L} \mid \mathrm{N} \& \mathrm{~h}=\mathrm{R}$ & $\mathrm{r}=\operatorname{Red}$ \\
\hline If $\mathrm{i}=\mathrm{B} \mid \mathrm{VB} \& \mathrm{~s}=\mathrm{L} \& \mathrm{~h}=\mathrm{R}$ & $r=$ Pink \\
\hline
\end{tabular}

Table 7. Refined inference rules

\begin{tabular}{ll}
\hline Condition & Conclusion \\
\hline If $\mathrm{i}=\mathrm{D}|\mathrm{B}| \mathrm{VB} \& \mathrm{~s}=\mathrm{N} \& \mathrm{~h}=\mathrm{R}$ & $\mathrm{r}=$ Red \\
If $\mathrm{i}=\mathrm{B} \mid \mathrm{VB} \& \mathrm{~s}=\mathrm{L} \& \mathrm{~h}=\mathrm{R}$ & $\mathrm{r}=$ Pink \\
\hline
\end{tabular}

\section{Experimental analysis and results}

\subsection{Comparison with conventional color recognition model}

There was no significant difference between the RGB model and the HSI model for color inference. Color inference is very subjective and abstract when setting appropriate values for specific intervals. However, we derived somewhat reliable results, as shown in Table 8, when the inference rules were simply further subdivided.

The conventional method[4] derived 64 inference rules by 4 separate RGB intervals and could be theoretically categorized into 64 colors. On the other hand, our method derived a total 144 inference rules for 12 kinds of Hue, 3 kinds of Saturation, 4 kinds of Intensity and could be theoretically categorized into 144 colors. The problems due to exponential inference rules should be accompanied by repetitive and efficient experiments to process pre-definition and improve confidence. However, our inference method appears to be more confident even without experimental confirmation. 


\begin{tabular}{llll}
\multicolumn{2}{l}{ Table 8. Inference results of the RGB and HSI models } \\
\hline RGB Value & HSI Value & Conclusion & \\
\cline { 3 - 4 } & & RGB & HSI \\
\hline $255,255,255$ & $0^{\circ}, 0,255$ & White & White \\
$204,204,255$ & $240^{\circ}, 0.2,255$ & Violet & Blue \\
$255,204,204$ & $0^{\circ}, 0.2,255$ & Gray & Pink \\
$255,255,204$ & $60^{\circ}, 0.2,255$ & Gray & Yellow \\
$204,255,204$ & $120^{\circ}, 0.2,255$ & Yellow-green & Green \\
$204,204,204$ & $0^{\circ}, 0,204$ & null & Gray \\
$102,153,255$ & $220^{\circ}, 0.6,255$ & Blue & Blue \\
$153,153,255$ & $240^{\circ}, 0.4,255$ & Violet & Blue \\
$153,102,255$ & $260^{\circ}, 0.6,255$ & Violet & Violet \\
$255,153,255$ & $300^{\circ}, 0.4,255$ & Violet & Purple \\
$255,153,153$ & $0^{\circ}, 0.4,255$ & Gray & Pink \\
$255,204,51$ & $45^{\circ}, 0,255$ & Orange & Yellow \\
$102,255,102$ & $120^{\circ}, 0.6,255$ & Yellow-green & Green \\
$51,255,153$ & $150^{\circ}, 0.8,255$ & Blue-green & Green \\
$51,255,204$ & $165^{\circ}, 0.8,255$ & Blue-green & Blue-green \\
$0,204,204$ & $180^{\circ}, 1.0,204$ & Blue-green & Blue-green \\
$0,51,255$ & $228^{\circ}, 1.0,255$ & Blue & Blue \\
$204,0,204$ & $300^{\circ}, 1.0,204$ & Violet & Purple \\
$153,0,51$ & $340^{\circ}, 1.0,153$ & Red & Pink \\
$153,51,0$ & $20^{\circ}, 1.0,153$ & Red & Orange \\
$97,62,0$ & $38^{\circ}, 1.0,97$ & Red & Brown \\
$0,0,102$ & $240^{\circ}, 1.0,102$ & Navy & Blue \\
$102,0,102$ & $300^{\circ}, 1.0,102$ & Violet & Purple \\
$0,51,51$ & $180^{\circ}, 1.0,51$ & Black & Blue-green \\
$51,102,0$ & $90^{\circ}, 1.0,102$ & Blue-green & Green \\
$102,204,0$ & $90^{\circ}, 1.0,204$ & Green & Yellow-green \\
$0,51,51$ & $180^{\circ}, 1.0,51$ & Black & Blue-green \\
\hline & & & \\
\hline
\end{tabular}

\subsection{Results of membership interval merger}

We applied the random input values $\mathrm{H}: 173^{\circ}$, S:1.0, I:255 for Cyan(C), which are unused intervals in Hue to check for derived inference rules due to the merging of membership intervals. Tables 9 and 10 show the results from applying 12 kinds of membership degrees for output in Hue using the center of gravity method.

\subsection{Results of add, modify, delete inference rules}

Ultimately, our proposed membership interval merger is to be used to handle inference rules at runtime. Conventional methods are not able to do this, whereas our method can freely handle inference rules, as shown in Table 11. We confirmed that the inference rules for Yellow, as defined in Table 5, convert to (H:Y, S:L, I:VB). The important point here is the definition method for the inference rules. We can see that the applied inference rules, such as "very bright(VB), $\operatorname{light}(\mathrm{L})$, yellow line(Y)" are defined by human perception. Because, the HSI model uses inference rules, it 
can intuitively change. In the conventional RGB model, it is difficult to analyze ordinary peoples perceptions of each RGB value for a specific color. Furthermore, if inference rules are changed by membership intervals that are obtained from a color that is simply selected, considerably abstract and unintended results could be derived by the user.

Table 9. Membership degree of individual intervals for Hue

\begin{tabular}{ll}
\hline Interval & Membership degree \\
\hline Red (R) & 0.0 \\
Red-Yellow (R-Y) & 0.0 \\
Yellow (Y) & 0.0 \\
Yellow-Green (Y-G) & 0.0 \\
Green (G) & 0.345800 \\
Green-Cyan (G-C) & 0.0 \\
Cyan (C) & 0.0 \\
Cyan-Blue (C-B) & 0.0 \\
Blue (B) & 0.0 \\
Blue-Magenta (B-M) & 0.0 \\
Magenta (M) & 0.0 \\
Magenta-Red (M-R) & 0.0 \\
\hline
\end{tabular}

Table 10. Membership degree of each color for inference results

\begin{tabular}{ll}
\hline Color & Membership degree \\
\hline Black & 0.0 \\
Yellow & 0.0 \\
Green & 0.345800 \\
Purple & 0.0 \\
Orange & 0.0 \\
Pink & 0.0 \\
Blue & 0.0 \\
Red & 0.0 \\
Brown & 0.0 \\
Gray & 0.0 \\
White & 0.0 \\
\hline
\end{tabular}

Input values in Table 11 are special values to output Yellow. Comparative analysis of Table 8 and Table 11 shows obvious differences to previously applied inference rules.

Analyzing the derived parts of Black in the results of Table 11, the three input values of the results represent the color to the yellow line. However, the reason for the derived results in Table 11 is that merging of the membership intervals did not take place, because of membership intervals of each input value are used all of them. Also, inference results depend on AND/OR fuzzy logic[2]; thus, the derived values of the inference results are all 0 . This problem needs to be resolved in the future.

The results for inference rule addition are shown in Table 12. Adding inference rules "very bright(VB), light(L), yellow line(Y)" for the color Beige to the defined inference rules in Table 5, 
we derived the results shown in Table 12 .

Table 11. Recognition results for variation of inference rules

\begin{tabular}{ll}
\hline Input value & Result \\
\hline $\mathrm{H}: 60^{\circ}, \mathrm{S}: 0.1, \mathrm{I}: 244$ & Yellow \\
$\mathrm{H}: 45^{\circ}, \mathrm{S}: 0.8, \mathrm{I}: 255$ & Orange \\
$\mathrm{H}: 60^{\circ}, \mathrm{S}: 1.0, \mathrm{I}: 255$ & Black \\
$\mathrm{H}: 60^{\circ}, \mathrm{S}: 1.0, \mathrm{I}: 153$ & Black \\
$\mathrm{H}: 80^{\circ}, \mathrm{S}: 0.6, \mathrm{I}: 255$ & Black \\
\hline
\end{tabular}

Table 12. Recognition results for inference rule addition

\begin{tabular}{ll}
\hline Input value & Result \\
\hline $\mathrm{H}: 60^{\circ}, \mathrm{S}: 0.1, \mathrm{I}: 244$ & Beige \\
$\mathrm{H}: 45^{\circ}, \mathrm{S}: 0.8, \mathrm{I}: 255$ & Yellow \\
$\mathrm{H}: 60^{\circ}, \mathrm{S}: 1.0, \mathrm{I}: 255$ & Yellow \\
$\mathrm{H}: 60^{\circ}, \mathrm{S}: 1.0, \mathrm{I}: 153$ & Yellow \\
$\mathrm{H}: 80^{\circ}, \mathrm{S}: 0.6, \mathrm{I}: 255$ & Yellow \\
\hline
\end{tabular}

As mentioned above, the results have different inference for H:60', S:0.1, I:244 as Yellow in Table 8 when the color Beige is not added to the inference rules. Thus, color information recognition by adding inference rules is efficient. Also, since the values for Hue, Saturation, and Intensity are similar to color recognition in humans, we could experimentally prove the efficiency of adding inference rules for specific colors.

\section{Conclusion}

In this paper, we propose an inference method based on the HSI model; color recognition in this method is similar to that by human beings. Our method allows the user to add, modify, or delete of inference rules.

We refined the inference rules by replacing the previously proposed RGB model with the HSI model and performed color information recognition by using partially defined inference rules that were added, modified, or deleted at runtime. Experimental results showed that our color recognition method is more intuitive and efficient for color information analysis and recognition than is the static inference method based on the conventional RGB model. However, the proposed method for merging membership intervals is unsuitable for some colors. Further research must be carried out to address the issue of membership intervals remaining unmerged under special conditions.

\section{Acknowledements}

This work was supported by a 2-Year Research Grant of Pusan National University. 


\section{References}

[1] Mohammad Jamshidi, Nader Vadiee, and Timothy J. Ross, editors. Fuzzy logic and control: software and hardware applications. Prentice-Hall, Inc., Upper Saddle River, NJ, USA, 1993.

[2] Abraham Kandel. Fuzzy Control Systems. CRC Press, Inc., Boca Raton, FL, USA, 1994.

[3] K.-B. Kim, G.-Y. Chae, and A.S. Pandya. Color preference and personality using fuzzy logic. International Journal of KIMICS, 2(1):32-35, 2004.

[4] K.-B. Kim and J.-H. Cho. A study on a sensitivity processing using a fuzzy reasoning rule. The Korea Society of Computer and Information, 12(3):1-8, 2007.

[5] K.-B. Kim and D.-H. Song. Nucleus recognition of uterine cervical pap-smears using fuzzy reasoning rule. The Korea Society of Computer and Information, 13(3):179-188, 2008.

[6] K.-B. Kim and Y.-W. Woo. Sensitivity analysis using color information and fuzzy rules. In Proceeding of International Conference of KIMIC, pages 91-94, 2009.

[7] George J. Klir and Bo Yuan, editors. Fuzzy Sets and Fuzzy Logic: Theory and Applications. Prentice Hall, Inc., Upper Saddle River, New Jersey., 1995.

[8] K.-S. Lee and H.-M. Chung. The emotion inference using differential of symbolic multiple valued logic functions. In Proceeding of KFIS Fall Conference, pages 493-496, 2002.

[9] M. Mizumoto. Fuzzy Controls under Product-Sum-Gravity Method and New Fuzzy Control Methods, pages 275294. CRC Press, Inc., 1993.

[10] Jiachuan Shi and Yutian Liu. Fuzzy evaluation based multi-objective reactive power optimization in distribution networks. Lecture Notes in Computer Science, 3613:11-19, 2005. 


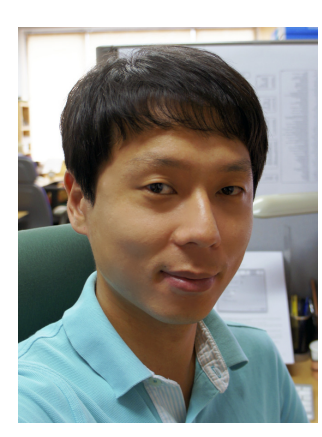

\section{Authors}

\section{Sang-Geol Lee}

He received the B.S. degree in Computer Science and Engineering from Pusan National University, Busan, Korea, in 2003, the M.S. degree in Computer Engineering from Pusan National University, Busan, Korea, in 2005. He is currently working toward the Ph.D. degree in Computer Engineering. His research interests include image processing, pattern recognition, neural networks, NPR, and fuzzy control.

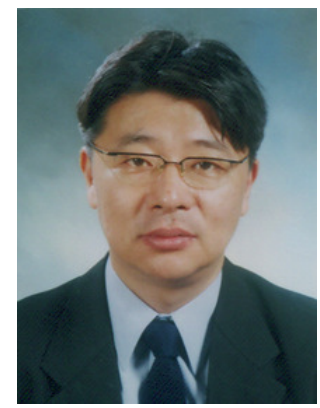

\section{Kwang-Baek Kim}

He received the M.S. and the Ph.D. degrees from Pusan National University, Busan, Korea, in 1993 and 1999, respectively. He is currently a professor in the Department of Computer Engineering, Silla University, Korea. His research interests include Fuzzy Neural Networks and Application, Biomedical Image Analysis, Image Processing, Biomedical system.

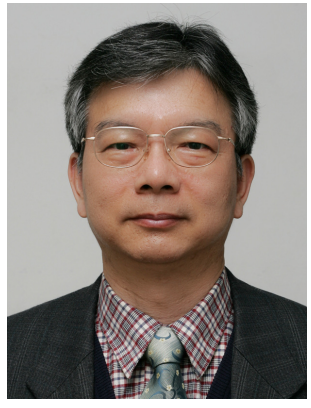

\section{Eui-Young Cha}

He received the B.S. degree in Electronic Engineering from Kyungpook National University, Daegu, Korea, in 1979, the M.S. degree in Computer Science from Seoul National University, Seoul, Korea, in 1982, the Ph.D degree in Computer Engineering from Seoul National University, Seoul, Korea, in 1998. From 1981 to 1985, he was a researcher of ETRI (Electronics and Telecommunications Research Institute), Daejeon, Korea. He was a Visiting Professor with the Faculty of Engineering, University of London, U.K. from 1995 to 1996 . He is currently a professor in Computer Engineering, Pusan National University, Busan, Korea. 
International Journal of Multimedia and Ubiquitous Engineering Vol.8, No.5 (2013) 